\title{
DULDUNG UND ZWANG BEIM UMGANG MIT NATURGEFAHREN UND RESSOURCEN IN DER STADT DER FRÜHEN NEUZEIT
}

\section{Die KLEINE EISZEIT UND IHRE AUSWIRKUNGEN AUF STÄDTISCHE GESELLSCHAFTEN}

Duldung und Zwang sind Aspekte des gesellschaftlichen Lebens, die es seit Beginn der Sesshaftigkeit des Menschen gegeben hat, wobei vor allem Zwang als Grundbedingung von Herrschaft und Verwaltung angesehen werden kann. Im folgenden Text soll in Form eines Werkstattberichts, der eine in die Tiefe gehende Beschäftigung mit dieser Thematik anregen will, das Verhältnis von Duldung (Toleranz) und Zwang anhand einiger Beispiele für die Stadt der Frühen Neuzeit, besonders des 17. und 18. Jahrhunderts, unter einem umweltgeschichtlichen Blickwinkel betrachtet werden ${ }^{1}$. Dies soll im Kontext der Kleinen Eiszeit geschehen, deren Auswir-

1 Zur Umweltgeschichte der Stadt in der Frühen Neuzeit vgl. einleitend: R. Reith, Umweltgeschichte der Frühen Neuzeit, (2011), S. 59-70 und 123-134 sowie U. Rosseaux, Städte in der Frühen Neuzeit, (2006), S. 94-114; zur Polizey, die die Verwaltungspraxis der Obrigkeit in der Frühen Neuzeit beschreibt, vgl. z. B. A. Iseli, Gute Policey. Öffentliche Ordnung in der Frühen Neuzeit, (2009); J. Bahlke, Landesherrschaft, Territorien und Staat in der frühen Neuzeit, (2012), S. 82-85 und A. Landwehr, „Normdurchsetzung“ in der Frühen Neuzeit? Kritik eines Begriffs, in: Zeitschrift für Geschichtswissenschaft, 48 (2000) sowie edierte Polizeyordnungen, z. B. in Die "gute" Policey im Reichskreis 6. Zur frühmodernen Normensetzung in den Kernregionen des Alten Reichs. Ein Quellenwerk. Policeyordnungen in den fränkischen Hochstiften Bamberg, Eichstätt und Würzburg, hg. v. W. Wüst, (2013). 
kungen einleitend kurz beschrieben werden. Anschließend untersucht ein erster Abschnitt Begriffsdeutungen von „Duldung" und "Zwang“ anhand von Lexika aus der zweiten Hälfte des 18. Jahrhunderts, die die zeitgenössischen Diskurse widerspiegeln und zusammenfassen. Danach folgen verschiedene Beispiele, die sich vorwiegend auf die Städte Utrecht, Braunschweig und Würzburg beziehen².

Die Frühe Neuzeit war zeitlich in weiten Teilen deckungsgleich mit der sogenannten Kleinen Eiszeit, einer Periode, in der es zwischen 1550 und $1850 \mathrm{zu}$ Temperaturrückgängen zwischen 1,5 und $2^{\circ} \mathrm{C}$ kam und die zum Teil regional, häufig jedoch auch in ganz Europa zu extremen Wetterlagen führten. Diese schlossen lange kalte Winter, feuchte kühle Sommer, Hochwasser, Unwetter, große Schneemengen auch im Flachland sowie langfristig das Wachstum von Gletschern im Gebirge und Verschiebungen der Vegetationsgrenzen ein ${ }^{3}$. Die Ursachen dieses langanhaltenden durchschnittlichen Temperaturrückgangs sind nicht vollständig geklärt. Vermutlich spielten dabei Veränderungen der Sonnenaktivität, eine verminderte Anzahl von Sonnenflecken, außerdem aber auch einige große Vulkanausbrüche im südlichen Pazifik und in Südamerika eine Rolle ${ }^{4}$. Die prägnantesten bildlichen Überlieferungen dieser Epoche sind niederländische Landschaftsbilder mit vereisten Wasserläufen, wie sie zum Beispiel Hendrick Avercamp Anfang des 17. Jahrhunderts malte 5 . Auf ihnen ist eine weitgehend positive Stimmung festgehalten, andere Bildquellen ge-

Für das 17. Jahrhundert zeigte Weber u.a. im Kontext von Naturgefahren die Dualität von Belohnungen und Strafen in Schriften der politischen Theorie, um bestimmte Verhaltensweisen der Untertanen zu beeinflussen; W. Weber, Prudentia gubernatoria. Studien zur Herrschaftslehre in der deutschen politischen Wissenschaft des 17. Jahrhunderts, (1992), S. 303-306.

${ }^{2}$ Die Auswahl der betrachteten Städte, die sich v. a. in ihrer Größe ähnelten, beruht dabei auf meinen bisherigen Forschungen im Rahmen des Göttinger Projekts Nachhaltigkeit als Argument (URL: www.umweltgeschichte.org, aufgerufen 27.10.2017).

${ }^{3}$ Hier und im Folgenden: R. Glaser, Kleine Eiszeit, in: Enzyklopädie der Neuzeit, hg. v. F. Jaeger, 6 (2007), Sp. 767-771; W. Behringer, Kulturgeschichte des Klimas. Von der Eiszeit bis zur globalen Erwärmung, (2007), S. 121-134.

${ }^{4}$ Behringer, Kulturgeschichte, S. 121-122.

5 Seine Bilder sind z. B. von der Londoner National Gallery im Internet zugänglich gemacht worden (URL: https://www.nationalgallery.org.uk/artists/hendrick-avercamp; aufgerufen 27.10.2017). 
ben jedoch die gravierenden negativen Folgen des kühlen Klimas wieder ${ }^{6}$. Infolge der klimatischen Veränderungen kam es, besonders während Temperaturminima wie dem Maunder-Minimum um 1700, zu Missernten, steigenden Preisen für Grundnahrungsmittel, einer verbreiteten Unterernährung der Bevölkerung und damit im Zusammenhang stehend $\mathrm{zu}$ einer größeren Anfälligkeit gegenüber Krankheiten wie der Pest, Typhus und Pocken ${ }^{7}$. Die Einflüsse der Kleinen Eiszeit auf kulturelle Phänomene wie die Malerei oder die Hexenverfolgung werden in der Forschung zum Teil kritisch gesehen ${ }^{8}$, sollen im Folgenden aber auch nicht weiter verfolgt werden. Die Städte der Frühen Neuzeit waren direkt oder indirekt ebenfalls intensiv von Naturereignissen betroffen: Stürme und Unwetter bedrohten Menschen und Gebäude in der Stadt, Hochwasser waren unter anderem für die Infrastruktur der Stadt gefährlich und erhöhten im Umland der Städte die Gefahr des Ausbruchs der Rinderseuche, und Missernten steigerten die Preise für Grundnahrungsmittel ${ }^{9}$, wodurch es häufig zu den erwähnten negativen Folgen kam.

\section{DULDUNG UND ZWANG IN DER FRÜHNEUZEITLICHEN STADT - BEGRIFFLICHKEITEN}

Duldung und Toleranz gelten als Synonyme, die grundsätzlich nicht nur auf religiöse Aspekte bezogen werden. Inhaltlich geht es vor allem um die Duldung von kultureller Andersartigkeit in einem Umfeld, das eine Mehrheit mit festgelegten Traditionen und Handlungsweisen besitzt. Im Verlauf der Neuzeit erweiterten sich zwar die Grenzen der Duldung bzw.

\footnotetext{
${ }^{6}$ Vgl. z. B. die Abbildung eines Bergrutsches, der die Stadt Plurs in Graubünden 1618 zerstörte; Behringer, Kulturgeschichte, S. 190.

7 Ebenda, S. 152-154; zur Entwicklung während dieser Zeit in England vgl. B. Fagan, The Little Ice Age. How Climate Made History, 1300-1850, (2002), S. 131-138.

8 C. Zwierlein, Klimageschichte und Kulturgeschichte der Frühen Neuzeit. Zum Problem des interdisziplinären Dialogs, in: Empirie in der Literaturwissenschaft, hg. v. C. Rauen, K. Mellmann, P. Ajouri, (2013), S. 344-348.

9 Vgl. dazu beispielsweise die durchschnittlichen jährlichen Getreidepreise zwischen 1636 und 1749 in Braunschweig bei T. Lassen, Hungerkrisen. Genese und Bewältigung von Hunger in ausgewählten Territorien Nordwestdeutschlands 1690-1750, (2016), S. 405.
} 
Toleranz (vor allem in religiösen Dingen), der moralische Standard breiter Gesellschaftsschichten war aber weiterhin die Intoleranz ${ }^{10}$.

Im Lexikon des Johann Heinrich Zedler, das zwischen 1731 und 1754 in Leipzig erschien, beschränkte sich die Toleranz, die auch als „Dultung“ bezeichnet wurde, vorrangig auf das Verhalten einer Obrigkeit, „welche in einer Provinz oder Stadt geschehen lässet, daß auch andere Religions-Verwandten ausser der daselbst eingeführten Religion, und welcher sie selbst zugethan ist, die freye Uebung ihres Gottesdienstes darinnen haben mö-

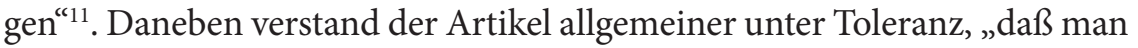
äusserlich im gemeinen Leben friedlich mit einander umzugehen suchet, einander die Pflichten des Rechts der Natur nicht versaget ${ }^{\text {"12. }}$. Wenn auch im Zedler „Duldung“ nicht als eigenes Stichwort aufgeführt ist, so gibt es dafür den Eintrag „Patientia“, der die „stillschweigende Einwilligung“ meint und vor allem im Rechtswesen verwendet wurde ${ }^{13}$. Ausführlicher behandelte ein Artikel das Stichwort „Zwang“"14. Dieser galt als Mittel, „wodurch die Wahl und Ausübung des Willens gehindert wird“, ohne jedoch den Willen der Person selbst zu brechen ${ }^{15}$.

Auch in der Enzyklopädie des Johann Georg Krünitz, die am Ende der Frühen Neuzeit entstanden war, gibt es einen eigenen ausführlichen Artikel zum Thema Toleranz ${ }^{16}$. Darin bezog Krünitz sich allein auf reli-

10 G. Eckert, Toleranz, in: Enzyklopädie der Neuzeit, hg. v. F. Jaeger, 13 (2011), Sp. 619; s. auch: J. und W. Grimm, Deutsches Wörterbuch, 2 (1854 und 1961), Sp. 1509, Stichwort: Duldung; vgl. zum Begriff der Toleranz und der Ausdehnung seines Gebrauchs auch: G. Besier, XI. ,Toleranz' als religionspolitischer Begriff im 17. und 18. Jahrhundert, in: Geschichtliche Grundbegriffe, hg. v. O. Brunner, W. Conze, R. Koselleck, 6 (2004), S. 495-510.

11 J.H. Zedler, Grosses vollständiges Universal-Lexicon aller Wissenschaften und Künste, 44 (1731-1754), Sp. 1115-1117. Der Artikel ist der Toleranz im Alltag gegenüber positiv eingestellt: „Die Erfahrung aber lehret uns täglich, daß Privat-Personen von unterschiedenen Meynungen, wenn sie sonst nur wollen, gantz friedlich und schiedlich bey einander leben und wohnen".Vgl. zu diesem, auch im Internet zugänglichen (URL: www. zedler-lexikon.de, aufgerufen 27.10.2017) Lexikon, das knapp 300.000 Artikel umfasst und elf definierte Wissenschaftsfelder wie z. B. Geographie und Handwerk behandelt: K. Lohsträter, F. Schock, Die gesammelte Welt. Studien zu Zedlers, Universal-Lexicon. Einführung, in: Die gesammelte Welt. Studien zu Zedlers, Universal-Lexicon', hg. v. dens., (2013), S. 2 , 7.

12 Zedler, Universal-Lexicon, 44, Sp. 1116.

13 Ebenda, 26, Sp. 1318.

14 Ebenda, 64, Sp. 963-987.

15 Ebenda, Sp. 978.

16 J. G. Krünitz, Oekonomische Encyklopädie, oder allgemeines System der Staats- 
giöse Toleranz, zwischen Christen, Muslimen und Juden sowie zwischen verschiedenen christlichen Konfessionen. Zwang wurde andererseits im selben Lexikon unter anderem beschrieben als: „3) die Nöthigung zu einem, der eigenen Neigung zuwiderlaufenden Thun oder Leiden; -- 4) (Rechtsw.), so v. v. Gewalt und zwar meist unrechtmäßige Gewalt; doch gehört zu dem gerechten Zwang besonders der Zwang im Staate, d. i. die von Seiten des Staates gegen die, welche den Zwecken des Staates zuwiderhandeln, angewendete Gewalt ${ }^{\text {"17 }}$. Zeitgenossen sahen daneben auch Zwänge wie den Zunftzwang innerhalb der städtischen Gesellschaften

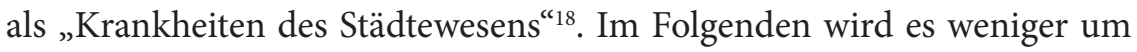
Toleranz im religiösen Sinn, sondern meist um Duldung oder Patientia von Obrigkeiten gegenüber abweichendem Verhalten von Untertanen gehen, die Anordnungen und Gesetze ignorierten. Daneben wird Zwang als obrigkeitliches Gebot im Rahmen der "guten Polizey“ verstanden ${ }^{19}$, der als legitimiert und gerecht erscheinende Hinderung des Willens anderer Personen eingesetzt wurde.

In diesem Sinn übten Obrigkeiten und Verwaltungen frühneuzeitlicher Städte Zwang aus, wenn Naturkatastrophen und Verknappungen bei der Versorgung mit Ressourcen (zum Beispiel Getreide) eintraten, die häufig mit der Kleinen Eiszeit in Zusammenhang standen. Es kam zu Geboten

Stadt- Haus- u. Landwirthschaft, in alphabetischer Ordnung 185, (1773-1858), S. 571-591; URL: http://www.kruenitz1.uni-trier.de/ (aufgerufen 27.10.2017); vgl. zu diesem Werk, das ursprünglich die Übersetzung einer französischen Enzyklopädie von 1770/1771 sein sollte und das Krünitz selbst bis zu seinem Tod 1796 bis zum Band 72 fortsetzte: D. Bouziane, H. Krems, R. Weiß, „... und die Lust und Trieb zu arbeiten unbeschreiblich... “. Johann Georg Krünitz und seine Oekonomisch-technologische Encyklopädie, (1996), S. 13-19. Der Eintrag „Duldung“ verweist direkt auf „Toleranz“ (Krünitz, Encyklopädie 9, S. 732). Eine klare Bezugnahme zur religiösen Toleranz findet sich auch in einem weniger ausführlichen Werk: J. Hübner, Reales Staats- Zeitungs- und Conversations-Lexicon, (1789), Sp. 2577-2578; URL: http://resolver.sub.uni-goettingen.de/purl?PPN683175858, aufgerufen am 27.10.2017.

17 Krünitz, Encyklopädie 242, S. 447.

18 M. Sandl, Ökonomie des Raumes. Der kameralwissenschaftliche Entwurf der Staatswissenschaft im 18. Jahrhundert, (1999), S. 42.

19 A. Iseli, Krisenbewältigung im 17. Jahrhundert. Die Rolle der guten Polizey, in: Die Krise in der Frühen Neuzeit, hg. v. R. Schlögl, P. R. Hoffmann-Rehnitz, E. Wiebel, (2016), S. 147-148. 
und Verboten in Form von gedruckten Verordnungen, die in der Regel ausgehängt und öffentlich verlesen wurden, und zu Reaktionen der betroffenen Untertanen.

\section{FRÜHNEUZEITLICHE STÄDTE ZWISCHEN OBRIGKEITLICHEM ZWANG UND DULDUNG}

Im Bereich des städtischen politischen und sozialen Handelns kann man davon ausgehen, dass Zwang vonseiten der Obrigkeit formal die Regel war. Duldung und Toleranz stellten dagegen Ausnahmen dar. An einigen Schlaglichtern aus dem 17. und 18. Jahrhundert sollen Praktiken von Duldung/ Toleranz und Zwang im Folgenden erörtert werden. Dargestellt werden dabei ein Sturm in Utrecht im Jahr 1674, der bis heute im kulturellen Gedächtnis der Stadt präsent ist, die Seuchengefahr in Braunschweig im 17. und 18. Jahrhundert, Nahrungskrisen des 18. Jahrhunderts in Braunschweig und Stadthagen sowie schließlich der kalte Winter mit dem darauffolgenden Hochwasser 1784 in Würzburg, dessen Markierung sogar am Rathaus ${ }^{20}$ dieser Stadt festgehalten wurde.

\subsection{EIN TORNADO IN UTRECHT 1674}

Charakteristisch für die Kleine Eiszeit waren große Sturmfluten an der Nordsee, wie sie zum Beispiel 1634 und 1717 vorkamen, und die viele Opfer, große Schäden und Landverluste bewirkten ${ }^{21}$. Auch ein Sturm über Land - inwieweit er konkret durch Veränderungen der Kleinen Eiszeit hervorgerufen wurde, sei einmal dahingestellt - konnte große Schäden

20 Der Eintrag zum Hochwasser von 1784 lautet: „A[nn]o 1784 den 29: Febr: ist der Mayn dieser Linie gleichgestanden“. Es wurde hier nur vom Magdalenhochwasser von 1342 übertroffen; vgl. dazu: V. Winiwarter, H.-R. Bork, Geschichte unserer Umwelt. Sechzig Reisen durch die Zeit, (2014), S. 20-21.

21 Vgl. M. Jakubowski-Tiessen, „Erschreckliche und unerhörte Wasserflut“. Wahrnehmung und Deutung der Flutkatastrophe von 1634, in: Um Himmels Willen. Religion in Katastrophenzeiten, hg. v. M. Jakubowski-Tiessen, H. Lehmann, (2003), S. 179-200; M. Jakubowski-Tiessen, Sturmflut 1717. Die Bewältigung einer Naturkatastrophe in der frühen Neuzeit, (1992). 
anrichten. Am 1. August 1674 raste ein Tornado ${ }^{22}$ von Frankreich kommend über Utrecht in den Niederlanden hinweg. Diese wohlhabende, calvinistisch geprägte Universitätsstadt und Hauptstadt der gleichnamigen niederländischen Provinz liegt ca. 40 Kilometer südöstlich von Amsterdam und besaß 1680 etwa 31.000 Einwohner ${ }^{23}$. Während des Kriegs zwischen den Niederlanden auf der einen und England, Frankreich und Münster auf der anderen Seite (1672-1674/1678) war Utrecht kurz zuvor von französischen Truppen besetzt gewesen (Juni 1672 bis November 1673) und musste enorme Kontributionen leisten ${ }^{24}$. Dieser verlustreichen Zeit folgte mit dem Tornado eine Naturkatastrophe, die die Stadt bis heute prägt. Innerhalb einer Viertelstunde kam es zu großen Zerstörungen. Beinahe alle Kirchen wurden beschädigt, das Mittelschiff des Doms stürzte sogar vollständig ein ${ }^{25}$.

Auch die Pieterskerk im östlichen Teil der Stadt wurde zerstört, so dass die dortige französische, katholische Gemeinde eine Ausweichmöglichkeit brauchte. Dabei muss berücksichtigt werden, dass sich in Utrecht seit dem Beginn des 17. Jahrhunderts ein Wandel im Umgang mit der katholischen Minderheit der Einwohner vollzog. Während anfangs katholische Messen durch städtische Bedienstete aktiv unterbunden wurden, konnten die Katholiken der Stadt, die um 1700 ein Drittel der Bevölkerung stellten und zu denen angesehene Bürger gehörten, ihren Gottesdienst später in versteckten Kirchen (schuilkerken) ausüben ${ }^{26}$. Nach dem Tornado folgte der calvinistische Kirchenrat dieser Linie. Er zeigte sich der katholischen Gemeinde gegenüber tolerant und bot ihr verschiedene andere Kirchen als Ersatz zur Auswahl an ${ }^{27}$. Da der Sturm allgemein als göttliches Strafgericht

22 Diese Form eines Unwetters nimmt ein Meteorologe an: J. F. den Tonkelaar, Het middenschip van de Dom vernietigd door een tornado?, in: Jaarboek Oud Utrecht (1980), S. 109.

23 A. H. M. van Schaik, Een nieuwe heer en een andere leer (1528-1618), in: ,Een paradijs vol weelde. Geschiedenis van de stad Utrecht, hg. v. R. E. de Bruin, (2000), S. 249.

24 D. E. A. Faber, R. Rommes, Op weg naar stabiliteit (1618-1747), in: ,Een paradijs vol weelde؛ Geschiedenis van de stad Utrecht, hg. v. R. E. de Bruin, (2000), S. 290-293, 296; vgl. dazu auch: H. Lademacher, Geschichte der Niederlande, (1983), S. 120-126.

25 D. E. A. Faber, R. Rommes, Op weg naar stabiliteit, S. 295.

26 A. H. M. van Schaik, Een nieuwe heer, S. 240-241; D. E. A. Faber, R. Rommes, Op weg naar stabiliteit, S. 256, 258.

27 A. Graafhuis, Het „schrickelijck tempeest“ van 1674, in: Jaarboek Oud Utrecht, (1974), S. 103-104. 
betrachtet wurde, verlangten Geistliche anschließend konsequent eine religiöse Umkehr der Bürger und die Verringerung der Sünden ${ }^{28}$. Dazu zählten auch die Überlegungen von Kirchenrat und Stadtrat, einen beschädigten Ballspielplatz im südlichen Teil der Stadt nicht wieder aufzubauen, da dort „durch die Spieler schon so oft der Tag und der Name des Herrn entheiligt wurden “29. Toleranz wurde somit gegenüber einer anderen christlichen Kirche geübt, Versuche von Zwangsmaßnahmen betrafen eher kirchenfernes Verhalten, das nicht toleriert werden sollte.

\subsection{SEUCHENGEFAHR IN BRAUNSCHWEIG}

Verbunden mit Missernten und Mangelernährung kam es generell zu einer Schwächung der menschlichen Immunsysteme, die dadurch anfälliger für Krankheiten und Seuchen wurden. In Braunschweig, das um 1671 etwa 15.500 und 1758 etwa 22.500 Einwohner besaß, war es $1657 \mathrm{zu}$ einem Ausbruch der Pest mit über 5.000 Toten gekommen ${ }^{30}$. Die Stadt war dabei als ehemaliger Vorort der Hanse und durch ihre geographische Lage ein Schnittpunkt von Fernhandelswegen aus allen Himmelsrichtungen und besaß bis 1671 Autonomie, als sie endgültig durch die welfischen Herzöge unter Rudolf August von Braunschweig-Wolfenbüttel erobert wurde ${ }^{31}$. Im Verlauf des 17. und 18. Jahrhunderts nahm die Anzahl von Seuchenaus-

28 Dieses straftheologische Argument stellte ein weit verbreitetes Phänomen dar. Vgl. J. van Eijnatten, F. van Lieburg, Niederländische Religionsgeschichte, (2011), S. 267; M. L. Allemeyer, Fewersnoth und Flammenschwert. Stadtbrände in der Frühen Neuzeit, (2007), S. 64.

29 A. Graafhuis, Het „schrickelijck tempeest", S. 105 [Übersetzung A.S.].

${ }^{30}$ N.-M. Pingel, Einwohnerzahlen, in: Braunschweiger Stadtlexikon, hg. v. L. Camerer, (1992), S. 66; R. Moderhack, Braunschweiger Stadtgeschichte. Mit Zeittafel und Bibliographie (1997), S. 118; D. Mack, Pest, in: Braunschweiger Stadtlexikon, hg. v. L. Camerer, (1992), S. 179. Zur Pest in der Frühen Neuzeit allgemein vgl. beispielsweise: F. Mauelshagen, Pestepidemien im Europa der Frühen Neuzeit (1500-1800), in: Pest. Die Geschichte eines Menschheitstraumas, hg. v. M. Meier, (2005), S. 237-265; M. Stolberg, Pestgestank und Hüttenrauch. Die Geschichte der Lufthygiene, in: „Sei sauber...!" Eine Geschichte der Hygiene und öffentlichen Gesundheitsvorsorge in Europa, (2004), S. 110-117 und C. C. Wahrmann, Kommunikation der Pest. Seestädte des Ostseeraums und die Bedrohung durch die Seuche 1708-1713, (2012). Zur großen Pest von 1665/1666 in London: E. Lord, The Great Plague. A people's history, (2014).

31 R. Moderhack, Braunschweiger Stadtgeschichte, S. 35-36; K. H. Kaufhold, Handel und Verkehrswesen, in: Die Wirtschafts- und Sozialgeschichte des Braunschweigischen 
brüchen im Vergleich zum Jahrhundert zuvor ab und die Maßnahmen der Obrigkeit konzentrierten sich auf die Abwehr von auswärtigen Krankheiten $^{32}$. Die Obrigkeit reagierte vorwiegend mit Zwangsmaßnahmen, die ambivalente Ziele verfolgten, auf die drohende Gefahr. Im Mai 1657 kam es zum wiederholten Mal zu einer Epidemie in der Stadt, und der Herzog von Braunschweig-Wolfenbüttel gab eine Verordnung heraus, nachdem „eine anklebende Säuche in Unserer Stadt BrunsWyk leyder immermeer

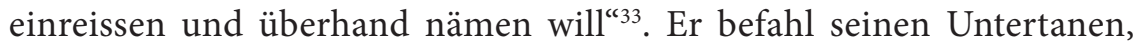
die Stadt, die sich einer direkten Unterwerfung unter den Herzog stets widersetzt hatte, nicht zu betreten und mit ihr keinen Handel zu treiben; gleichermaßen verbot er den Bürgern, die Stadt zu verlassen oder mit dem Umland Handel zu treiben. Für den Herzog bedeutete die Seuche somit einerseits eine Gefahr, da sie sich in seinem Territorium ausbreiten konnte, andererseits bot sie sich als Vorwand für eine Strafmaßnahme gegen die rebellische Stadt an. Tatsächlich sahen sich die Bürger der Stadt gezwungen, innerhalb der Mauern zu bleiben. Ein Tagebucheintrag in Knittelversform von 1657 gibt dies deutlich wieder:

Dar hier die pestelenzi war/

Da mussen wir alle gemein/

von unsern nachbarrn belagertt sein

Dar mussen reiter undt bauren

auff unsser burger lauren

wen wir unss teten hinaus wagen

wurden wir baltt zuruck geschlagen

wen ettliche burger kamen hinaus

Landes, 2: Frühneuzeit, hg. v. C. Märtl u. a. (2008), S. 671; D. Diestelmann, Kleine Braunschweiger Stadtgeschichte, (2010), S. 76.

32 Um 1750 soll es in Nordwestdeutschland zum letzten Ausbruch der Pest gekommen sein; N. Bulst, Vier Jahrhunderte Pest in niedersächsischen Städten. Vom Schwarzen Tod (1349-1351) bis in die erste Hälfte des 18. Jahrhunderts, in: Stadt im Wandel. Kunst und Kultur des Bürgertums in Norddeutschland 1150-1650, hg. v. C. Meckseper, (1985), S. 257.

33 Hier und im Folgenden: Stadtarchiv (weiter: StadtA) Braunschweig, B I 25, Nr. 16, Bl. 95 . 
wollte niemantt sie herbergen im hauß

wen wir kamen in ihr lantt

wurden wir gedan in den bantt $t^{34}$.

Am 10. April 1658 hob der Herzog von Braunschweig-Wolfenbüttel die Verordnung vom Vorjahr wieder auf, nachdem „solche Säuche sich gestillet hat", und ließ auch die Ausrichtung von Jahrmärkten erneut zu, ohne dass er jedoch die Kontrolle über die Stadt übernehmen konnte ${ }^{35}$. Als Vorsichtsmaßnahme gegen ein erneutes Auftreten der Seuche sollten unter anderem in Braunschweig alle Gegenstände, die verdächtigt wurden, die Krankheit zu übertragen, nicht transportiert und verkauft werden. Dazu gehörten vor allem Gegenstände aus Wolle, Leinen und Pelz, somit auch Bettzeug und Kleider, die ansonsten konfisziert und verbrannt oder vergraben werden sollten. Der Wortlaut der Verordnung lässt wenig Spielraum für die Duldung von individuellem Vorgehen, da selbst das Verschweigen von verbotenen Verkäufen der genannten Gegenstände bestraft werden sollte $^{36}$. Es ist aber durchaus fraglich, ob diese Anordnungen in der Praxis stets umgesetzt werden konnten, da kurzfristige Einnahmen im alltäglichen Kampf um das Überleben für Einzelne sehr wichtig waren ${ }^{37}$.

Als Reaktion auf Nachrichten aus anderen Gebieten über den Ausbruch von ansteckenden Krankheiten kam es zu Handelssperren und zur Isolation gegenüber den betroffenen Regionen. Im späten 17. Jahrhundert betraf dies auch die nähere Umgebung mit Orten wie Nordhausen, Duderstadt oder Stendal, Anfang des 18. Jahrhunderts verstärkt weiter entfernte Gegenden

34 StadtA Braunschweig, H V Nr. 150, o. D.

35 StadtA Braunschweig, H V Nr. 150, Drucksachen.

36 „Wan über kurtz oder lang kund werden sollte / dass jemand Wissenschaft von solchen heimlichen oder öffentlich ein oder durchgefürten Geräte gehabt / sol derselbe mit swerer Straffe unnachlässig beleget werden“, ebenda.

37 „Man könnte meinen, daß im Angesicht der Pest eine Interessengleichheit vorherrsche und damit kein Durchsetzungsproblem [von Verordnungen] bestünde [...]. Aber der Schein täuscht, denn die Motivationen dahinter waren jeweils andere. Der Obrigkeit oder Regierung ging es um den Schutz eines Territoriums als Ganzes (oder des noch nicht betroffenen Teiles), seiner Wirtschaft und insbesondere des Handels; den Untertanen um die Lage vor Ort, um ihre ganz persönliche Situation oder die ihrer Stadt“. O. Ulbricht, Einleitung. Die Allgegenwärtigkeit der Pest in der Frühen Neuzeit und ihre Vernachlässigung in der Geschichtswissenschaft, in: Die leidige Seuche. Pest-Fälle in der Frühen Neuzeit, hg. v. O. Ulbricht, (2004), S. 44. 
wie Polen oder Südosteuropa ${ }^{38}$. In der zweiten Hälfte des 18. Jahrhunderts brachte dann die Diskussion um die medizinischen Vorsorgemaßnahmen gegen die Pocken, die in eine Zeit fielen, als Regierungen auch über Ärzte und die medizinische Polizei die Kontrolle über ihre Territorien verstärken wollten ${ }^{39}$, eine Abkehr vom bisherigen Wechselspiel von Duldung und Zwang. Die sogenannte Inokulation gegen die Pocken wurde ursprünglich im Osmanischen Reich angewendet und gelangte Anfang des 18. Jahrhunderts zuerst nach England, wurde ab den 1760er Jahren in Österreich, Frankreich und Russland angewendet, führte durch unsachgemäße Ausführung aber häufig zu Ausbrüchen der Krankheit ${ }^{40}$. Anders als in den Jahrzehnten zuvor versuchten die Braunschweiger Behörden im Fall der Impfungen die Untertanen ohne direkten Zwang zu überzeugen (zum Beispiel über Veröffentlichungen in den Braunschweigischen Anzeigen $)^{41}$. Das Hauptproblem stellte dabei vermutlich das Risiko dar, das mit der Inokulation einherging. Obwohl der Herzog, der Geheime Rat und ein Teil der Obrigkeit ihre Kinder behandeln ließen bzw. diese Praktik befürworteten, zögerten die Untertanen ${ }^{42}$. Erst als um 1800 mit dem Übergang zur sicheren

38 StadtA Braunschweig, H V Nr. 150, Edikte Herzog Rudolf August v. 22.12.1681, 19.6.1682, 7.8.1682 sowie StadtA Braunschweig C I 1, Nr. 13, Seuchensperre gegen Schlesien, Polen, Preußen und Pommern v. 25.8.1710.

39 T. Broman, Zwischen Staat und Konsumgesellschaft: Aufklärung und die Entwicklung des deutschen Medizinalwesens im 18. Jahrhundert, in: Zwischen Aufklärung, Policey und Verwaltung. Zur Genese des Medizinalwesens 1750-1850, hg. v. B. Wahrig, W. Sohn, (2003), S. 91-92. Zur theoretischen Fundierung: C. Möller, Medizinalpolizei. Die Theorie des staatlichen Gesundheitswesens im 18. und 19. Jahrhundert, (2005). Zur Pockeninokulation mit Argumenten dafür und dagegen, ebd., S. 29-30.

40 S. Winkle, Geißeln der Menschheit. Kulturgeschichte der Seuchen, (1997), S. 868-880. Ein früher Praktiker der Inokulation war der Altonaer Arzt Johann Friedrich Struensee (1737-1772), der auch die Ausbreitung der Krankheit durch Ansteckung erkannte und die Isolation von Erkrankten anordnete; zu der Furcht vor lebenslanger Zeichnung durch die Pocken vgl.: M. Stolberg, Homo patiens. Krankheits- und Körpererfahrung in der Frühen Neuzeit, (2003), S. 73.

41 Vgl. Braunschweigische Anzeigen, 94, 97, 99 (1755), Sp. 1881-1891, 1945-1953; 1985-1990. Auf verschiedene Bedenken wird dabei im zweiten Teil (97, Sp. 1974) mit Gegenargumenten geantwortet, z. B.: „Sind die eingepfropften Blattern auch ein sicheres Bewahrungsmittel gegen die natürlichen? Antwort. Die Erfahrung widerlegt diesen Einwurf am besten. Seit 30. Jahren hat man kein einziges erwiesenes Beyspiel, daß eine oculirte Person die Blattern zum zweytenmale bekommen hätte. Die Feinde der Einpfropfung nehmen ihre Zuflucht zum Betruge."

42 P. Albrecht, Von den vergeblichen Mühen, die Bevölkerung der Stadt Braunschweig 
Kuhpockenimpfung das Risiko reduziert worden war, nahm die Akzeptanz des Vorgangs in der Bevölkerung $\mathrm{zu}^{43}$. Zwangsmaßnahmen fanden sich demnach im Braunschweiger Beispiel bis zum Anfang des 18. Jahrhunderts im Bereich der Abwehr der Pest zum Beispiel durch Isolierung der Stadt. Gegen Ende des Jahrhunderts setzte die Obrigkeit dagegen im Fall der Pockeninokulation auf die Überzeugung der Untertanen.

\subsection{NAHRUNGSKRISEN IN BRAUNSCHWEIG UND STADTHAGEN} IM 18. JAHRHUNDERT

Nahrungskrisen entstanden nicht allein aufgrund von schlechten Ernten, sondern konnten auch durch fehlende Zugangsrechte, Preisspekulationen und Hindernisse beim Transport von Lebensmitteln in einzelnen Städten oder Territorien entstehen ${ }^{44}$. In der Frühen Neuzeit reagierte die Obrigkeit darauf mit einem verhältnismäßig gleichbleibenden Repertoire an Maßnahmen, das Michael Huhn als Kanon der Teuerungsmaßnahmen bezeichnete ${ }^{45}$. Dazu gehörte u.a. die Errichtung sogenannter Korn- oder Fruchtsperren, die den Abfluss von Getreide aus einem Territorium in das Ausland verhindern und die Versorgung der eigenen (Stadt-) Bevölkerung sicherstellen sollte.

In Braunschweig lassen sich die deutlichsten Nahrungskrisen des 18. Jahrhunderts in den Jahren 1720, 1756-1758 und 1770-1772 verorten.

von der Nützlichkeit des Impfens gegen Blattern zu überzeugen (1754-1787), in: Gotts verhengnis und seine straffe. Zur Geschichte der Seuchen in der Frühen Neuzeit, (2005), S. $128-129$.

43 Ebd., S. 135; die Kuhpockenimpfung wurde vom englischen Landarzt Edward Jenner in den 1790er Jahren erprobt und eingeführt, S. Winkle, Geißeln der Menschheit, S. 880-901. Medizinische, aber auch nichtmedizinische Beweggründe der Bevölkerung gegen die Pockenimpfung hat Wolff für Württemberg zusammengetragen; E. Wolff, Einschneidende Maßnahmen. Pockenschutzimpfungen und traditionelle Gesellschaft im Württemberg des frühen 19. Jahrhunderts, (1998), S. 296-446.

44 Vgl. dazu einen aktuellen Sammelband mit weiterführender Literatur und Fallbeispielen aus verschiedenen Weltregionen: Famines During the ,Little Ice Age (1300-1800). Socionatural Entanglements in Premodern Societies, hg. v. D. Collet, M. Schuh, (2017); eine grundlegende Arbeit zu diesem Thema bildet weiterhin: W. Abel, Massenarmut und Hungerkrisen im vorindustriellen Europa. Versuch einer Synopsis, (1974).

45 M. Huhn, Ein Ernstfall des Konsums. Obrigkeitliche Teuerungspolitik im Übergang zur Moderne, in: Der lange Weg in den Überfluss. Anfänge und Entwicklung der Konsumgesellschaft seit der Vormoderne, hg. v. M. Prinz, (2003), S. 233-234. 
1720 wurde es Händlern verboten, Getreide um die Stadt herumzuführen ${ }^{46}$. In den 1750ern wurden daraufhin der Vorkauf von Getreide auf dem Land und der Ankauf durch Auswärtige sowie die Ausfuhr untersagt, aber besonders weiter entfernt wohnende Amtmänner und Pächter hielten sich wenig daran und verkauften das Getreide entsprechend den eigenen Interessen. Konzessionierte Händler durften auch weiterhin frei handeln, und da eine große Zahl der Getreideverkäufer dem Landadel angehörte, wurden deren Verstöße gegen herzogliche Verordnungen nachsichtig behandelt ${ }^{47}$. In den 1770er Jahren, als eine mehrere Länder betreffende Nahrungskrise auch Braunschweig betraf, wurde hier erneut die Kornausfuhr verboten. Dabei versuchten die umfassenden Bestimmungen der Verordnung, Löcher zu schließen, die zuvor von den Untertanen genutzt worden waren. So gab es Ausflüchte, vorjähriges Korn sei nicht von der Sperre betroffen und dass der Verkauf der Früchte bereits vor dem Erlass der Verordnung vereinbart worden $\operatorname{sei}^{48}$. Die Händler versuchten demnach, eine Duldung ihrer Praktiken $\mathrm{zu}$ erreichen, die Obrigkeit dagegen ihre Anordnungen durchzusetzen ${ }^{49}$. Als Resultat lässt sich festhalten, dass damit schließlich „private Handelsberechtigungen und -freiheiten [...] dem allgemeinen Nutzen" weichen mussten $^{50}$. Zwangsmaßnahmen wie Preisfestsetzungen und Zwangsverkauf sollten auch gegenüber Gutspächtern eingesetzt werden, die „nichts, oder nicht nach Proportion ihres Vorraths, von Woche zu Woche, auf hiesigen Markt bringen lassen " ${ }^{\text {"51 }}$. Grundsätzlich versuchte die herzogliche Regierung allerdings, Kornsperren zu vermeiden, weil der freie Handel sich günstig auf die Versorgung der eigenen Bevölkerung auswirkte: „man solle die

46 T. Dehesselles, Policey, Handel und Kredit im Herzogtum Braunschweig-Wolfenbüttel in der frühen Neuzeit, (1999), S. 70.

47 P. Albrecht, Die Förderung des Landesausbaues im Herzogtum Braunschweig-Wolfenbüttel im Spiegel der Verwaltungsakten des 18. Jahrhunderts (1671-1806), (1980), S. 98, 101; T. Dehesselles, Policey, S. 72, 74.

48 P. Albrecht, Die Förderung, S. 103.

49 Man war „bestrebt, die Sperre auch wirklich durchzuführen. Es ergingen allerlei Ermahnungen, die Grenzsicherung und die Ausstellung von Pässen wurde Soldaten übertragen und zum Besuch ausländischer Mühlen mußten vorher obrigkeitliche Pässe gelöst werden, da sonst der Unterschleif gar zu groß sei“. Ebenda, S. 104.

\footnotetext{
50 T. Dehesselles, Policey, S. 74.

51 Braunschweigische Anzeigen, 28 (11.1.1772), Sp. 52.
} 
Untertanen anhalten, in die eigenen Städte zu liefern. Sperren könnten nur der letzte Ausweg sein" ${ }^{\text {" } 2}$.

Auch in der kleinen Grafschaft Schaumburg-Lippe, zwischen Minden und Hannover gelegen, gab es in der ersten Hälfte des 18. Jahrhunderts Klagen über Nahrungsmangel und Vorkäufe. Hier duldete der Fürst vorerst jedoch noch den freien Handel und setzte Zwangsmaßnahmen nur zögerlich ein. Lokale Obrigkeiten des kleinen Landes erbaten dagegen mehrfach die Einrichtung von Verboten des Vorkaufs und der Getreideausfuhr, um die eigene Versorgung zu sichern. So baten Bürgermeister und Rat von Stadthagen, einer unter dem Kommissariat eines gräflichen Stadtvogts stehenden, aber auch als Kurbad geförderten Stadt ${ }^{53}$ am 20. Oktober 1734, „nicht nur den höchst verderblichen Vorkauff bey hoher Straffe überhaupts, sondern auch besonders anbey die Ausfuhr des Korns sub poena confiscationis gnädigst verbiethen zu lassen ${ }^{\text {" } 54}$. Hier gelangten vermutlich Wünsche aus der Bevölkerung über die städtischen Behörden an die Landesherrschaft und anschließend wurden Normen ausgehandelt ${ }^{55}$. Zwangsmaßnahmen wie Kornsperren oder Preisfestsetzungen, die teilweise auch von der Bevölkerung gefordert wurden, standen somit in diesem Bereich der obrigkeitlichen Duldung von Gesetzesverstößen durch Getreidehändler gegenüber.

\subsection{EIS UND HOCHWASSER IN WÜRZBURG 1784}

Würzburg, die Residenz- und Hauptstadt des gleichnamigen Fürstbistums, stand spätestens seit dem 16. Jahrhundert unter der direkten Herrschaft des Bischofs, besaß 1772 etwa 15.000 Einwohner und war durch seine Lage am Main häufig Hochwasser ausgesetzt ${ }^{56} .1784$ kam es nun im mit-

52 T. Dehesselles, Policey, S. 71.

${ }^{53}$ F. Bartels, Stadthagen. Einst und jetzt, (1972), S. 32.

54 Zit. nach: T. Lassen, Hungerkrisen, S. 308.

55 A. Landwehr, „Normdurchsetzung“, S. 156.

56 H. Drüppel, Ratsverfassung und städtisches Gerichtswesen, in: Geschichte der Stadt Würzburg, hg. v. U. Wagner, 2 (2004), S. 235-240; H. Schott, Fürstlicher Absolutismus und barocke Stadt, in: Geschichte der Stadt Würzburg, hg. v. U. Wagner, 2 (2004), S. 158; R. Glaser, W. Schenk, Aspekte der vorindustriellen Umweltgeschichte des Würzburger Siedlungsraums, in: Geschichte der Stadt Würzburg, hg. v. U. Wagner, 2 (2004), S. 34, Abb. 1 (Hochwasserereignisse 1342-1784). 
teleuropäischen Flusssystem an vielen Orten zu Hochwasserereignissen, besonders am Rhein und seinen Zuflüssen sowie an der Elbe ${ }^{57}$. Dies hing direkt mit den vorherigen Monaten zusammen. Im besonders kalten Winter 1783/1784 waren die Flüsse vereist, zum Beispiel fror der Neckar bei Mannheim am 15. Dezember $1783 \mathrm{zu}$, und es fiel in den daran angrenzenden Gebieten viel Schnee; mit Schmelzwetter stiegen die Pegel der Flüsse an und erreichten Ende Februar / Anfang März 1784 ihren Höhepunkt. Am Main kam es außer in Würzburg auch in Bamberg, Kitzingen, Miltenberg, Hanau und Frankfurt zu Überschwemmungen, die u.a. Brücken und Kräne zerstörten ${ }^{58}$. Auch in der Stadt Würzburg gab es Mitte Februar 1784 viel Eis und Schnee; die Regierung des Hochstifts Würzburg verwarnte die Bürger der Stadt, da diese viel Unrat (vermutlich vor allem Abwasser und Fäkalien) auf die Straßen warfen, der dort zu einer Gefahr wurde ${ }^{59}$. Die Obrigkeit zwang die Untertanen jedoch nicht dazu, Eis und Schnee auf eigene Kosten zu entfernen, was möglicherweise aufgrund mangelnder Durchsetzungsfähigkeit geschah ${ }^{60}$. Bischof Franz Ludwig von Erthal stellte dafür Geld zur Verfügung, und die Hofkammer sollte Pferde zum Abtransport bereithalten. Am 27. Februar begann das Eis schließlich zu brechen: Eisschollen, Trümmer, Bäume und Geäst trieben durch die Stadt ${ }^{61}$. Aufgrund des Hochwassers wurde der Ausbruch von Seuchen befürchtet

57 Vgl. hier und im Folgenden: J. Buisman, Duizend jaar weer, wind en water in de Lage Landen, 1751-1800, 6 (2015), S. 636-651. Vgl. ausführlicher u.a. für Bonn: E. Ennen, Eisgang und Hochwasser von 1784 am Niederrhein, in: Stadtzerstörung und Wiederaufbau. Zerstörung durch Erdbeben, Feuer und Wasser, 1 (1999), S. 303-308.

58 J. Buisman, Duizend jaar weer, S. 637.

59 Hier und im Folgenden: H. Schott, Das Hochwasser von 1784, in: Geschichte der Stadt Würzburg, hg. v. U. Wagner, 2 (2004) S. 37-39.

60 Auch in anderen Bereichen wurden herrschaftliche Verordnungen in Würzburg nicht durchgesetzt. Hinsichtlich des Getreidehandels vgl. H. Schott, Das Verhältnis der Stadt Würzburg zur Landesherrschaft im 18. Jahrhundert, (1995), S. 404-406.

61 Vgl. auch: Curt Weikinn. Quellentexte zur Witterungsgeschichte Europas von der Zeitenwende bis zum Jahr 1850. Hydrographie, hg. v. M. Börngen, G. Tetzlaff, (2000), S. 389. Ein anonymer zeitgenössischer Druck beschreibt die Landschaft an den Flüssen: „Am Mayn, weiter am Rhein hinunter, u. am Necker, sieht man nichts, als die kläglichsten Verwüstungen. Wände ohne Dach, ungeheure Eisberge, halbeingerißene Häuser, Schiffe zu oberst gekehrt, abgeworfene Dächer, Gebäude ohne Thüren u. Fenster, halb zwischen dem Eiß hervorstehendes erstarrtes Vieh, einen Fluß voll Eiß gestepft, u. geflüchtete Einwohner in dem größten Elend“; Anom., Gründliche Nachricht derer so merkwürdigen Wasser-Fluthen, [1784]. 
und die betroffenen Kirchen wurden nach Absprache mit der geistlichen Regierung geschlossen. Die Holzhändler, deren losgerissene Stämme viele Schäden verursacht hatten, zum Beispiel an der Mainbrücke, dem Wehr, der unteren Mainmühle und an der Kranenmauer, mussten an die Hofkammer 5.000 Gulden Entschädigung bezahlen. Inwieweit die Aufräumarbeiten, die sich teilweise noch über vier Jahre hinzogen, freiwillig oder unter Zwang erfolgten, ist unklar. Die Stadt Würzburg übernahm jedenfalls nicht sämtliche Verantwortung und weigerte sich zum Beispiel noch 1788, auf eigene Kosten die durch die Überschwemmung am Mainloch aufgehäuften Steine zu entfernen ${ }^{62}$ und sah hier vermutlich erneut die Holzhändler in der Pflicht ${ }^{63}$. In Würzburg wurden demnach in diesem Fall die Untätigkeit der Bürger geduldet, die Holzhändler jedoch als Schadensverursacher zu Zahlungen gezwungen.

\section{FAZIT UND BRÜCKENSCHLAG IN DIE GEGENWART}

Duldung und Zwang, in geringerem Maß auch Toleranz, lassen sich in den oben beschriebenen kurzen Fallbeispielen im Kontext der klimatischen Ungunstphase der Kleinen Eiszeit beobachten. Das Verhältnis der beiden Reaktionsoptionen kann zwar an dieser Stelle nicht abschließend bestimmt werden, eine Vielzahl von verschiedenen Praktiken ist aber anzunehmen. Dies wird häufig durch die große Anzahl normativer Quellen überdeckt, durch die auch hinsichtlich Naturgefahren und der Nutzung und Versorgung mit Ressourcen ausführliche Bestimmungen in Kraft gesetzt wurden. Eingesetzt wurden diese Normen als ein zentrales Instrument der herrschaftlichen Legitimation (Braunschweig), als Mittel gegen negative Auswirkungen von Naturgefahren (zum Beispiel der Pest), teilweise, wie am Beispiel Stadthagens sichtbar, auf Wunsch der Bevölkerung. Daneben konnten natürlich auch religiöse Aspekte eine Rolle spielen, wie sie sich im calvinistisch geprägten Utrecht gegenüber einer Stätte des sportlichen Vergnügens zeigten.

${ }^{62}$ H. Schott, Das Hochwasser, S. 39.

63 Das Mainloch bezeichnete dabei den dritten Brückenbogen der Würzburger Mainbrücke, der bei Bedarf zum Flößen geöffnet wurde. C. G. Scharold, Würzburg und seine Umgebungen. Ein Wegweiser und Erinnerungsbuch, (1836), S. 66. 
Duldung war, entsprechend der in Zedlers Lexikon verwendeten Form der Patientia, meist informeller Art und spiegelte häufig wirtschaftliche und politische Machtverhältnisse und Aushandlungsprozesse zwischen den Akteuren wider. In Würzburg zeigte sich die bischöfliche Verwaltung teilweise nicht durchsetzungsfähig, in Braunschweig konnten zum Beispiel adelige Getreidehändler auch während einer Nahrungskrise weiter Getreide aufkaufen und ausführen, außerdem nutzten die Untertanen juristische Schlupflöcher, um ihre Interessen zu verfolgen.

Abschließend lassen sich drei allgemeinere Thesen formulieren, die den beschriebenen Themenkomplex betreffen:

- Angesichts naturaler Herausforderungen, die teilweise im Zusammenhang mit den klimatischen Veränderungen der Kleinen Eiszeit standen, war religiöse Toleranz beobachtbar und nicht allein eine Frage von Machtverhältnissen; sie war jedoch vom politischen, wirtschaftlichen und gesellschaftlichen Umfeld abhängig.

- Im Umgang mit Naturgefahren, Naturkatastrophen und knappen Ressourcen, zum Beispiel bei Nahrungskrisen, spiegelten die Durchsetzung von Zwangsmaßnahmen und die Duldung von Praktiken jedoch in der Regel die existierenden Machtverhältnisse in einer Stadt wider.

- Viele Zwangsmaßnahmen behielten über Jahrzehnte ihre inhaltliche Form; so war die Isolation sowohl im 17. als auch im 18. Jahrhundert das zentrale Schutzmittel gegen Epidemien.

Auch im 21. Jahrhundert spielen klimatische Veränderungen und der damit verbundene Meeresspiegelanstieg für viele Städte weltweit eine große Rolle ${ }^{64}$. Parallel dazu kommt es bei zentralen naturalen Ressourcen zu Engpässen ${ }^{65}$, so dass politische Aushandlungsprozesse, die heute jedoch auf globaler Ebene stattfinden müssen, Gegenmaßnahmen und Nutzungsrechte festlegen sollten. Auch bei diesen Prozessen spielen wie in der Frühen Neuzeit Zwang und Duldung von staatlicher Seite eine wichtige Rolle für gesellschaftliche Veränderungen.

64 Vgl. z. B.: Adapting Cities to Climate Change. Understanding and Addressing the Development Challenges, hg. v. J. Bicknell, D. Dodman, D. Satterthwaite, (2009).

${ }^{65}$ Vgl. u. a.: Non-Renewable Resource Issues. Geoscientific and Societal Challenges, hg. v. R. Sinding-Larsen, F.-W. Wellmer, (2012). 


\section{TOLEROWANIE I PRZYMUS W OBCHODZENIU SIĘ Z NATURALNYMI ZAGROŻENIAMI ORAZ ZASOBAMI W MIEŚCIE OKRESU WCZESNONOWOŻYTNEGO}

\section{STRESZCZENIE}

Artykuł bada, opierając się na sytuacji w różnych miastach Europy Środkowej (przede wszystkim w Brunszwiku, Würzburgu i Utrechcie), związek tolerowania i przymusu ze strony władz w odniesieniu do danej ludności miejskiej w kontekście środowiska naturalnego. Temat ten jest przedstawiany na tle klęsk żywiołowych i niedoborów zasobów, które częściowo wynikały z klimatycznych wpływów tzw. małej epoki lodowcowej (XV-XIX wiek).

Tłumacznie

Renata Skowrońska

\section{DULDUNG UND ZWANG BEIM UMGANG MIT NATURGEFAHREN UND RESSOURCEN IN DER STADT DER FRÜHEN NEUZEIT}

ZUSAMMENFASSUNG

Der vorliegende Aufsatz untersucht anhand von verschiedenen Städten Mitteleuropas (vor allem Braunschweig, Würzburg, Utrecht) den Zusammenhang von Duldung und Zwang von Seiten der Obrigkeit gegenüber der jeweiligen Stadtbevölkerung in einem umweltgeschichtlichen Kontext. Dies geschieht vor dem Hintergrund von Naturkatastrophen und Ressourcenknappheiten, die teilweise auf klimatische Einflüsse der sog. Kleinen Eiszeit (15.-19. Jahrhundert) zurückzuführen sind.

\section{ABIDANCE AND COERCION IN DEALING WITH NATURAL HAZARDS AND RESOURCES IN THE TOWN OF THE EARLY MODERN PERIOD}

\section{SUMMARY}

The article addresses the relation between abidance and coercion on the part of the authorities in relation to the population of a given town (mainly Braunschweig, Würzburg and Utrecht) in the context of the natural environment. This problem is presented against natural hazards and shortages of resources, which were partly determined by the climatic influence of the so called Little Ice Age (the $15-19^{\text {th }}$ centuries). 


\section{SŁOWA KLUCZOWE / SCHLAGWORTE / KEYWORDS}

- Nowożytność; historia miast; historia środowiska naturalnego; policja; klęski żywiołowe

- Frühe Neuzeit; Stadtgeschichte; Umweltgeschichte; Policey; Naturkatastrophen

- early modern period; history of cities; environmental history; police; natural disasters

\section{BIBLIOGRAFIA / BIBLIOGRAFIE / BIBLIOGRAPHY}

\section{ŹRÓDŁA ARCHIWALNE / ARCHIVALISCHE QUELLEN / ARCHIVAL SOURCES}

Stadtarchiv Braunschweig

- Sign. B I 25, Nr. 16, Städtische Verordnungen 1653-1660;

- Sign. C I 1, Nr. 13, Herzogliche Verordnungen 1710-1714;

- Sign. H V Nr. 150, Krankheiten.

\section{ŹróDłA DRUKOWANE / GEDRUCKTE QUELLEN / PRINTED SOURCES}

[Anonym], Gründliche Nachricht derer so merkwürdigen Wasser-Fluthen, [1784].

Braunschweigische Anzeigen, (1755 und 1772).

Hübner J., Reales Staats- Zeitungs- und Conversations-Lexicon, (1789).

Krünitz J. G., Oekonomische Encyklopädie, oder allgemeines System der Staats- Stadt-

Haus- $u$. Landwirthschaft, in alphabetischer Ordnung, (1773-1858).

Zedler J. H., Grosses vollständiges Universal-Lexicon aller Wissenschaften und Künste, (1731-1754).

\section{LITERATURA / LITERATUR / LITERATURE}

Abel W., Massenarmut und Hungerkrisen im vorindustriellen Europa. Versuch einer Synopsis, (1974).

Adapting Cities to Climate Change. Understanding and Addressing the Development Challenges, hg. v. J. Bicknell, D. Dodman, D. Satterthwaite, (2009).

Albrecht P., Von den vergeblichen Mühen, die Bevölkerung der Stadt Braunschweig von der Nützlichkeit des Impfens gegen Blattern zu überzeugen (1754-1787), in: Gotts verhengnis und seine straffe. Zur Geschichte der Seuchen in der Frühen Neuzeit, (2005), S. 127-137.

Albrecht P., Die Förderung des Landesausbaues im Herzogtum Braunschweig-Wolfenbüttel im Spiegel der Verwaltungsakten des 18. Jahrhunderts (1671-1806), (1980).

Allemeyer M. L., Fewersnoth und Flammenschwert. Stadtbrände in der Frühen Neuzeit, (2007).

Bahlke J., Landesherrschaft, Territorien und Staat in der frühen Neuzeit, (2012).

Bartels F., Stadthagen. Einst und jetzt, (1972).

Behringer W., Kulturgeschichte des Klimas. Von der Eiszeit bis zur globalen Erwärmung, (2007).

Besier G., XI. ,Toleranz' als religionspolitischer Begriff im 17. und 18. Jahrhundert, in: 
Geschichtliche Grundbegriffe, hg. v. O. Brunner, W. Conze, R. Koselleck, 6 (2004), S. 495-510.

Bouziane D., H. Krems, R Weiß, „... und die Lust und Trieb zu arbeiten unbeschreiblich...." Johann Georg Krünitz und seine Oekonomisch-technologische Encyklopädie, (1996).

Broman T., Zwischen Staat und Konsumgesellschaft: Aufklärung und die Entwicklung des deutschen Medizinalwesens im 18. Jahrhundert, in: Zwischen Aufklärung, Policey und Verwaltung. Zur Genese des Medizinalwesens 1750-1850, hg. v. B. Wahrig, W. Sohn, (2003), S. 91-107.

Buisman J., Duizend jaar weer, wind en water in de Lage Landen, 1751-1800, 6 (2015).

Bulst N., Vier Jahrhunderte Pest in niedersächsischen Städten. Vom Schwarzen Tod (1349-1351) bis in die erste Hälfte des 18. Jahrhunderts, in: Stadt im Wandel. Kunst und Kultur des Bürgertums in Norddeutschland 1150-1650, hg. v. C. Meckseper, (1985), S. 251-270.

Curt Weikinn. Quellentexte zur Witterungsgeschichte Europas von der Zeitenwende bis zum Jahr 1850. Hydrographie, hg. v. M. Börngen, G. Tetzlaff, (2000).

Dehesselles T., Policey, Handel und Kredit im Herzogtum Braunschweig-Wolfenbüttel in der frühen Neuzeit, (1999).

Diestelmann D., Kleine Braunschweiger Stadtgeschichte, (2010).

Die „gute" Policey im Reichskreis 6. Zur frühmodernen Normensetzung in den Kernregionen des Alten Reichs. Ein Quellenwerk. Policeyordnungen in den fränkischen Hochstiften Bamberg, Eichstätt und Würzburg, hg. v. W. Wüst, (2013).

Drüppel H., Ratsverfassung und städtisches Gerichtswesen, in: Geschichte der Stadt Würzburg, hg. v. U. Wagner, 2 (2004), S. 232-253.

Eckert G., Toleranz, in: Enzyklopädie der Neuzeit, hg. v. F. Jaeger, 13 (2011), Sp. 619-629.

Eijnatten J. van, Lieburg F. van, Niederländische Religionsgeschichte, (2011).

Ennen E., Eisgang und Hochwasser von 1784 am Niederrhein, in: Stadtzerstörung und Wiederaufbau. Zerstörung durch Erdbeben, Feuer und Wasser, 1 (1999), S. 303-308.

Faber D. E. A., Rommes R., Op weg naar stabiliteit (1618-1747), in: ,Een paradijs vol weelde. Geschiedenis van de stad Utrecht, hg. v. R. E. de Bruin, (2000), S. 251-313.

Fagan B., The Little Ice Age. How Climate Made History, 1300-1850, (2002).

Famines During the Little Ice Age' (1300-1800). Socionatural Entanglements in Premodern Societies, hg. v. D. Collet, M. Schuh, (2017).

Glaser R., Kleine Eiszeit, in: Enzyklopädie der Neuzeit, hg. v. F. Jaeger, 6 (2007), Sp. 767-771.

Glaser R., W. Schenk, Aspekte der vorindustriellen Umweltgeschichte des Würzburger Siedlungsraums, in: Geschichte der Stadt Würzburg, hg. v. U. Wagner, 2 (2004), S. 21-36.

Graafhuis A., Het „schrickelijck tempeest“ van 1674, in: Jaarboek Oud Utrecht, (1974), S. $96-107$.

Grimm J. und W., Deutsches Wörterbuch, 2 (1854 und 1961), Sp. 1509, Stichwort: Duldung.

Huhn M., Ein Ernstfall des Konsums. Obrigkeitliche Teuerungspolitik im Übergang zur Moderne, in: Der lange Weg in den Überfluss. Anfänge und Entwicklung der Konsumgesellschaft seit der Vormoderne, hg. v. M. Prinz, (2003), S. 233-234.

Iseli A., Krisenbewältigung im 17. Jahrhundert. Die Rolle der guten Polizey, in: Die Krise in der Frühen Neuzeit, hg. v. R. Schlögl, P. R. Hoffmann-Rehnitz, E. Wiebel, (2016), S. $147-167$. 
Iseli A., Gute Policey. Öffentliche Ordnung in der Frühen Neuzeit, (2009).

Jakubowski-Tiessen M., „Erschreckliche und unerhörte Wasserflut". Wahrnehmung und Deutung der Flutkatastrophe von 1634, in: Um Himmels Willen. Religion in Katastrophenzeiten, hg. v. M. Jakubowski-Tiessen, H. Lehmann, (2003), S. 179-200.

Jakubowski-Tiessen M., Sturmflut 1717. Die Bewältigung einer Naturkatastrophe in der frühen Neuzeit, (1992).

Kaufhold K. H., Handel und Verkehrswesen, in: Die Wirtschafts- und Sozialgeschichte des Braunschweigischen Landes. Band II Frühneuzeit, hg. v. C. Märtl u.a., (2008), S. 671-673.

Lademacher H., Geschichte der Niederlande, (1983).

Landwehr A., „Normdurchsetzung“ in der Frühen Neuzeit? Kritik eines Begriffs, in: Zeitschrift für Geschichtswissenschaft, 48 (2000), S. 146-162.

Lassen T., Hungerkrisen. Genese und Bewältigung von Hunger in ausgewählten Territorien Nordwestdeutschlands 1690-1750, (2016).

Lohsträter K., Schock F., Die gesammelte Welt. Studien zu Zedlers, Universal-Lexicon. Einführung, in: Die gesammelte Welt. Studien zu Zedlers, Universal-Lexicon', hg. v. dens., (2013), S. 1-18.

Lord E., The Great Plague. A people's history, (2014).

Mack D., Pest, in: Braunschweiger Stadtlexikon, hg. v. L. Camerer, (1992), S. 179.

Mauelshagen F., Pestepidemien im Europa der Frühen Neuzeit (1500-1800), in: Pest. Die Geschichte eines Menschheitstraumas, hg. v. M. Meier, (2005), S. 237-265.

Moderhack R., Braunschweiger Stadtgeschichte. Mit Zeittafel und Bibliographie, (1997).

Möller C., Medizinalpolizei. Die Theorie des staatlichen Gesundheitswesens im 18. und 19. Jahrhundert, (2005).

Non-Renewable Resource Issues. Geoscientific and Societal Challenges, hg. v. R. Sinding-Larsen, F.-W. Wellmer, (2012).

Pingel N.-M., Einwohnerzahlen, in: Braunschweiger Stadtlexikon, hg. v. L. Camerer, (1992), S. 66.

Reith R., Umweltgeschichte der Frühen Neuzeit, (2011), S. 1-18.

Rosseaux U., Städte in der Frühen Neuzeit, (2006).

Sandl M., Ökonomie des Raumes. Der kameralwissenschaftliche Entwurf der Staatswissenschaft im 18. Jahrhundert, (1999).

Schaik A. H. M. van, Een nieuwe heer en een andere leer (1528-1618), in:, Een paradijs vol weelde. Geschiedenis van de stad Utrecht, hg. v. R. E. de Bruin, (2000), S. 191-249.

Scharold C. G., Würzburg und seine Umgebungen. Ein Wegweiser und Erinnerungsbuch, (1836).

Schott H., Fürstlicher Absolutismus und barocke Stadt, in: Geschichte der Stadt Würzburg, hg. v. U. Wagner, 2 (2004), S. 130-202.

Schott H., Das Hochwasser von 1784, in: Geschichte der Stadt Würzburg, hg. v. U. Wagner, 2 (2004) S. 37-39.

Schott H., Das Verhältnis der Stadt Würzburg zur Landesherrschaft im 18. Jahrhundert, (1995).

Stolberg M., Pestgestank und Hüttenrauch. Die Geschichte der Lufthygiene, in: „Sei sauber...!" Eine Geschichte der Hygiene und öffentlichen Gesundheitsvorsorge in Europa, (2004), S. 110-117. 
Stolberg M., Homo patiens. Krankheits- und Körpererfahrung in der Frühen Neuzeit, (2003).

Tonkelaar J. F. den, Het middenschip van de Dom vernietigd door een tornado?, in: Jaarboek Oud Utrecht, (1980), S. 95-109.

Ulbricht O., Einleitung. Die Allgegenwärtigkeit der Pest in der Frühen Neuzeit und ihre Vernachlässigung in der Geschichtswissenschaft, in: Die leidige Seuche. Pest-Fälle in der Frühen Neuzeit, hg. v. O. Ulbricht, (2004), S. 1-63.

Wahrmann C. C., Kommunikation der Pest. Seestädte des Ostseeraums und die Bedrohung durch die Seuche 1708-1713, (2012).

Weber W., Prudentia gubernatoria. Studien zur Herrschaftslehre in der deutschen politischen Wissenschaft des 17. Jahrhunderts, (1992).

Winiwarter V., Bork H.-R., Geschichte unserer Umwelt. Sechzig Reisen durch die Zeit, (2014).

Winkle S., Geißeln der Menschheit. Kulturgeschichte der Seuchen, (1997).

Wolff E., Einschneidende Maßnahmen. Pockenschutzimpfungen und traditionelle Gesellschaft im Württemberg des frühen 19. Jahrhunderts, (1998).

Zwierlein C., Klimageschichte und Kulturgeschichte der Frühen Neuzeit. Zum Problem des interdisziplinären Dialogs, in: Empirie in der Literaturwissenschaft, hg. v. C. Rauen, K. Mellmann, P. Ajouri, (2013), S. 331-351.

\section{STRONY INTERNETOWE / INTERNETSEITEN / WEBSITES}

National Gallery, London, URL: https://www.nationalgallery.org.uk/artists/hendrick-avercamp (aufgerufen 27.10.2017).

Projekt Nachhaltigkeit als Argument, URL: www.umweltgeschichte.org (aufgerufen 27.10.2017). 


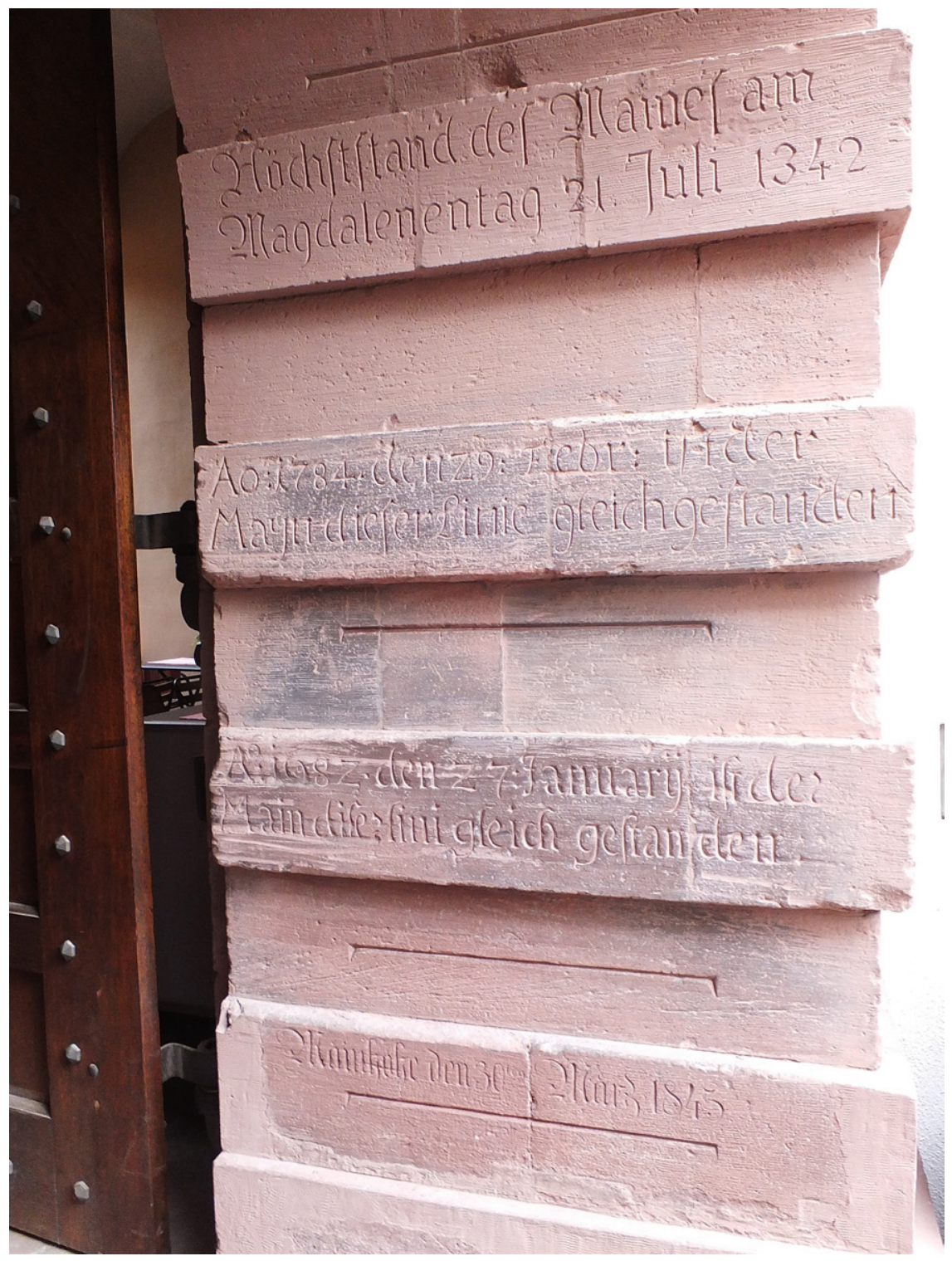

Bild 1. Hochwassermarken am Würzburger Rathaus (Foto: A. Schanbacher) 


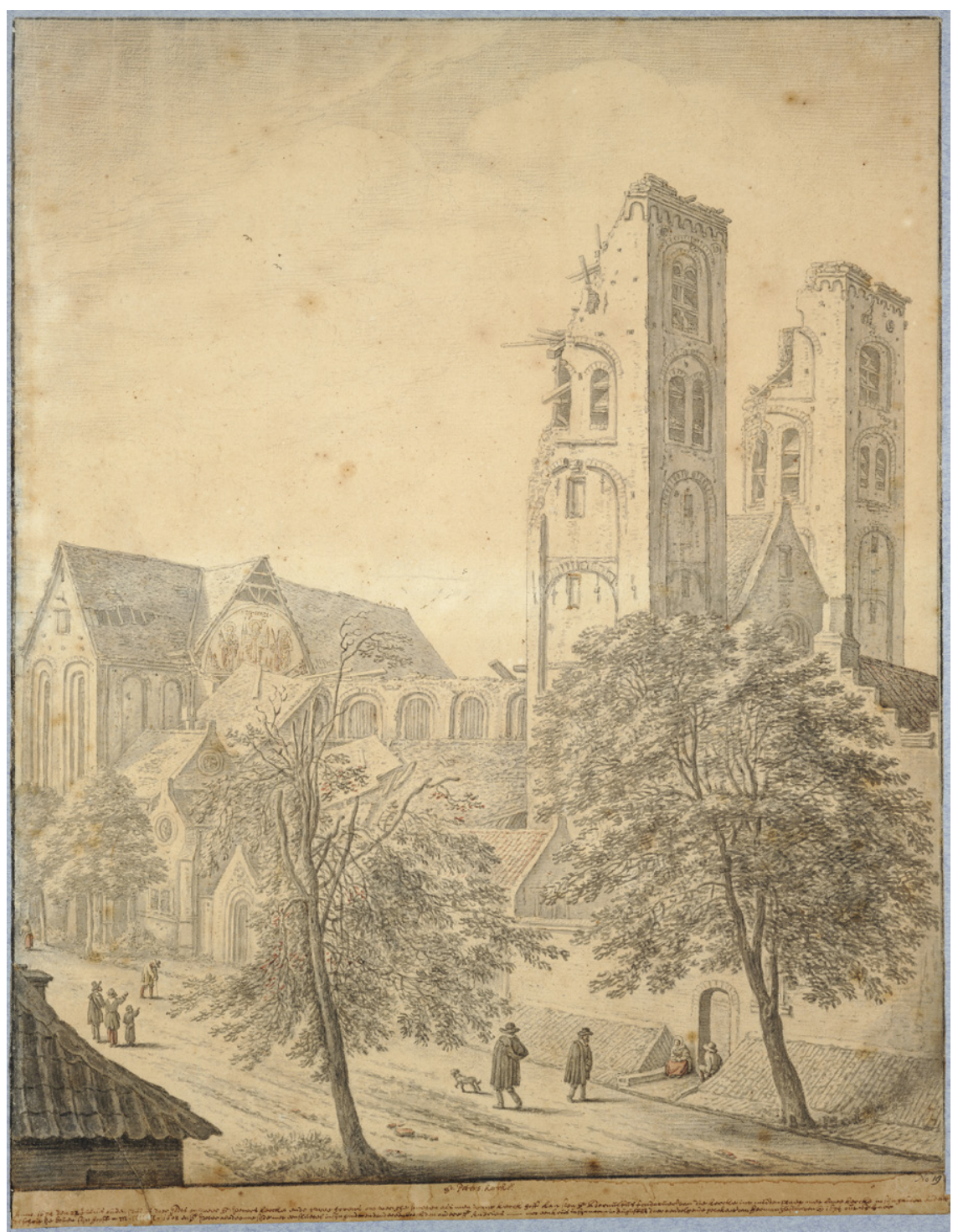

Bild 2. Die zerstörte Pieterskerk 1674 (Het Utrechts Archief, Bildersammlung, Nr. 28644) 